\title{
Communication
}

\section{Caenorhabditis elegans mom-4 is required for the activation of the p38 MAPK signaling pathway in the response to Pseudomonas aeruginosa infection}

\author{
Ajing $\mathrm{Xu}^{1,3^{*}}$, Guojun $\mathrm{Shi}^{3,4^{*}}$, Feng $\mathrm{Liu}^{3,4}$, Baoxue $\mathrm{Ge}^{2,3 \bowtie}$ \\ ${ }^{1}$ Department of Pharmacology, Xinhua Hospital, School of Medicine, Shanghai 200092, China \\ ${ }^{2}$ Shanghai pulmonary hospital, Tongji University School of Medicine, Shanghai 200092, China \\ ${ }^{3}$ Lab of Signal Transduction, Institute of Health Sciences, Shanghai Institutes for Biological Sciences, Chinese Academy of \\ Sciences and Shanghai Jiao-Tong University School of Medicine, Shanghai 200025, China \\ ${ }^{4}$ Graduate School of the Chinese Academy of Sciences, Beijing 100049, China \\ $\triangle$ Correspondence: gebaoxue@sibs.ac.cn \\ Received August 13, 2012 Accepted November 5, 2012
}

\begin{abstract}
The p38 mitogen-activated protein kinase (MAPK) plays an evolutionarily conserved role in the cellular response to microbial infection and environmental stress. Activation of $\mathrm{p} 38$ is mediated through phosphorylation by upstream MAPKK, which in turn is activated by MAPKKK. In the Caenorhabditis elegans, the p38 MAPK (also called PMK-1) signaling pathway has been shown to be required in its resistance to bacterial infection. However, how different upstream MAP2Ks and MAP3Ks specifically contribute to the activation of PMK-1 in response to bacterial infection still is not clearly understood. By using double-stranded RNA-mediated interference (RNAi) and genetic mutants of $C$. elegans, we demonstrate that $C$. elegans MOM-4, a mammalian TAK1 homolog, is required for the resistance of $C$. elegans to a $P$. aeruginosa infection. We have also found that the MKK-4 of $C$. elegans is required for $P$. aeruginosa resistance, but not through the regulation of DLK-1. In summary, our results indicate that different upstream MAPKKKs or MAPKKs regulate the activation of PMK-1 in response to $P$. aeruginosa.
\end{abstract}

\footnotetext{
KEYWORDS C. elegans, MAPK, innate immunity, p38, $P$. aeruginosa $\mathrm{PA}-14, \mathrm{MOM}-4$
}

\section{INTRODUCTION}

Mitogen-activated protein kinase (MAPK) signaling pathways play an important role in diverse physiological processes, including development, growth and proliferation, stress responses, and immunity (Herskowitz et al.,1995; Waskiewicz et al.,1995; Schaeffer et al.,1999; Widmann et al.,1999; Kyriakis et al., 2001). MAPK signal transduction pathways are conserved from yeast to mammals (Herskowitz et al.,1995; Waskiewicz et al., 1995; Widmann et al.,1999). Based on their structural and biochemical features, three main subgroups of MAPK have been identified, including extracellular signal-regulated kinase (ERK), stress-activated protein kinase (SAPK)/c-Jun N-terminal kinase (JNK) and p38/MPK2 (Cobb et al., 1991; Dérijard et al., 1994; Han et al., 1994; Kyriakis et al., 1994; Lee et al., 1994). Mammalian p38 has been shown to play an extremely important role in the immune system (Symons et al., 2006). Activation of p38 is regulated by upstream MAP2Ks, MKK3 and MKK6 (Raingeaud et al., 1996; Wysk M et al., 1999; Tanaka et al., 2002). Recent reports from loss-of-function studies demonstrate that MKK4 is also required for ultraviolet radiation-induced activation of p38 MAPK (Brancho et al., 2003). At higher upstream levels, numerous MAP3Ks including MEKK, ASK, TAK1, Tpl-2/Cot and MLK/DAK have been implicated in the p38 activation (Yamaguchi et al., 1995; Tibbles et al.,1996; Ichijo et al.,1997; Deacon et al.,1999; Chiariello et al., 2000). Our

\footnotetext{
*These authors contributed equally to the work. 
previous results have pointed out the complexity of the MAP kinase signaling pathway, which is due to the fact that each individual MAP kinase can be activated by two or three upstream MAP2Ks and multiple MAP3Ks in Drosophila under different stimuli (Zhuang et al., 2006). Upon activation, p38 can phosphorylate and activate a number of transcription factors including ATF-2/ATF-7, CHOP/GADD153, Elk1, MEF2-C, Sap1 and CREB (Wang et al.,1996; Raingeaud et al.,1996; Han et al.,1997; Iordanov et al.,1997; Whitmarsh et al.,1997; Shivers et al., 2010), and protein kinases such as MAPKAPK2, MNK, PRAK, MSK1 and RSK-B (Stokoe et al.,1992; Fukunaga et al.,1997; Waskiewicz et al.,1997; New et al.,1998; Deak et al.,1998; Pierrat et al.,1998), thus eliciting different cellular responses.

The nematode Caenorhabditis elegans has been used as a model host for infection studies with the human opportunistic pathogen Pseudomonas aeruginosa PA-14 to identify evolutionarily conserved mechanisms of innate immune responses (Kim et al., 2005). The mammalian p38 homolog PMK-1 has been found to be required for the resistance of $C$. elegans to $P$. aeruginosa infection (Berman et al., 2001; Kim et al., 2002). Further studies showed that the NSY-1/SEK-1/PMK-1 cassette is an evolutionarily conserved module used in the defense against $P$. aeruginosa infection (Kim et al., 2002). However, whether other MAP3Ks or MAP2Ks also contribute to the activation of PMK-1, remains unknown. On the other hand, although the
PMK-1 MAPK signaling pathway has been shown to play an important role in the innate immune response, little is known about whether the activation of PMK-1 is also involved in $C$. elegans's responses to other stimuli. In this study, we have used RNA interference (RNAi) and genetic mutants to suppress the expression of individual MAP kinases. Our results indicate that individual MAPKKKs and MAPKKs specifically regulate $\mathrm{PMK}-1$ activation.

\section{RESULTS}

Activation of $C$. elegans p38/PMK-1 by different stimuli

In C. elegans, the mammalian p38 homolog PMK-1 has been found to be required for the resistance to $P$. aeruginosa infection. To analyze the signaling pathway of the $C$. elegans PMK-1/p38 activation in other stresses, we first determined whether PMK-1 can be activated by different stimuli including heat shock, osmotic stress by Sorbitol, $P$. aeruginosa infection and UV stress. Wild type $C$. elegans strain N2 worms were exposed to different stimuli. The activation of PMK-1 was identified using p38 $\mathrm{T}^{180} / \mathrm{Y}^{182}$ phospho-specific antibody. As shown in Figures $1 \mathrm{~A}-\mathrm{D}$, the stimulation of $N 2$ worms with heat shock $\left(35^{\circ} \mathrm{C}\right)$, sorbitol $(200$ $\mathrm{mmol} / \mathrm{L}), P$. aeruginosa infection or UV $(1200 \mathrm{~J})$ distinctly induced the activation of PMK-1. These results suggest that PMK-1/p38 may play a general role in $C$. elegans's response to various stimuli.
A

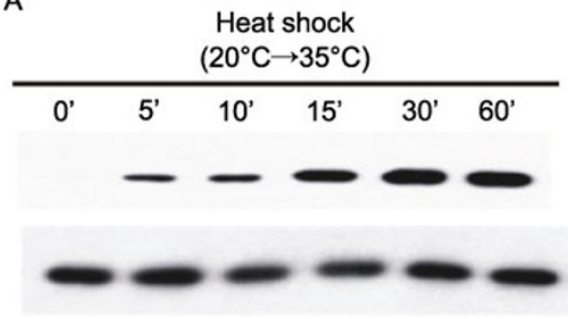

C

Pathogen stress

(P. aeruginosa PA14 infection)

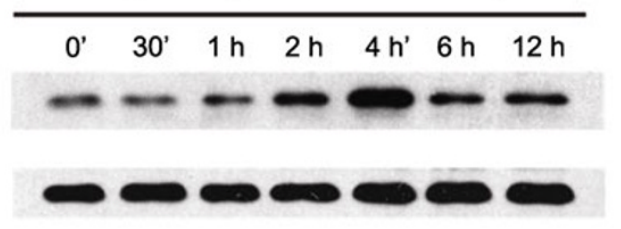

B

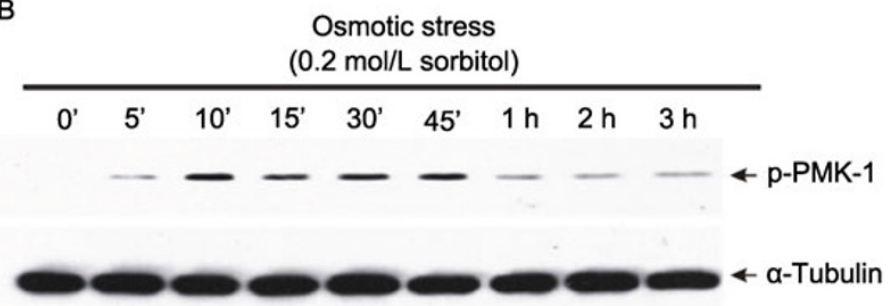

D

DNA damage stress

(UV radiation $1200 \mathrm{~J}$ )

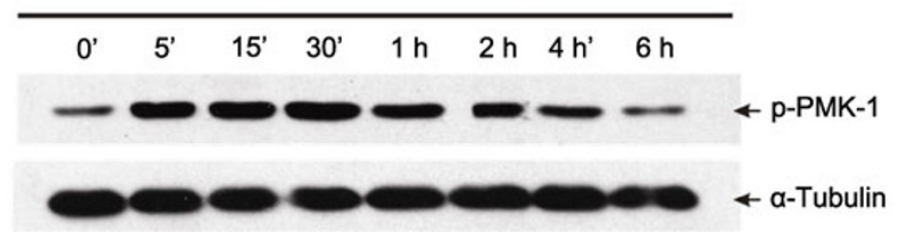

Figure 1. Activation of PMK-1 in C. elegans by various stimuli. (A) Heat shock. Young adult N2 worms were washed with M9 buffer from OP50-1 NGM plates, and incubated in a $35^{\circ} \mathrm{C}$ water bath for the time indicated. (B) Osmotic stress. Sorbitol was added to young adult $N 2$ worms in M9 buffer for a final concentration of $200 \mathrm{mmol} / \mathrm{L}$, and incubated for the time indicated. (C) Pathogenic infection. Young adult N2 worms were placed onto plates seeded with $P$. aeruginosa for the time indicated. (D) DNA damage stress. Young adult N2 worms were exposed to $1200 \mathrm{~J} \mathrm{UV}$, and then cultured in M9 buffer for the time indicated. Following exposure, worms were collected in M9 buffer, lysed and analyzed by western blot. Activation of PMK-1 was detected by blotting with anti-phospha-p38 antibody (upper panel), as indicated. Total proteins were measured by blotting with anti- $\alpha$-tubulin (lower panel). 
Activation of PMK-1 is required for resistance to $P$. aeruginosa infection

Previous studies have revealed that the activation of the NSY $-1 \rightarrow$ SEK-1 $\rightarrow$ PMK-1 MAP kinase cascade is essential for $C$. elegans to respond to pathogenic attacks (Kim et al., 2002).

To investigate further whether the different components of the PMK-1 signaling pathway play a role in the innate immune responses to microbial infection, we examined the survival rate of the MAP2K or MAP3K mutant strains after having $C$. elegans infected with the pathogenic $P$. aeruginosa strain $P$. aeruginosa. In consistence with previous findings, the $n s y-1$, sek-1 and pmk-1 mutant strains are more susceptible to $P$. aeruginosa infection (Fig. 2A). We have utilized RNAi to reduce the expression of $n s y-1$, and sek-1, and analyzed the susceptibility of these RNAi-treated worms to pathogenic $P$. aeruginosa infection (Fig. S1A). As shown in Fig. $2 \mathrm{~B}$, the RNAi of $n s y-1$, and sek-1 make $C$. elegans more susceptible to $P$. aeruginosa infection. Consistent with these results, RNAi of $n s y-1$, and sek-1 inhibited the activation of p38/PMK-1 in response to PA14 infection (Fig. S1B). The A

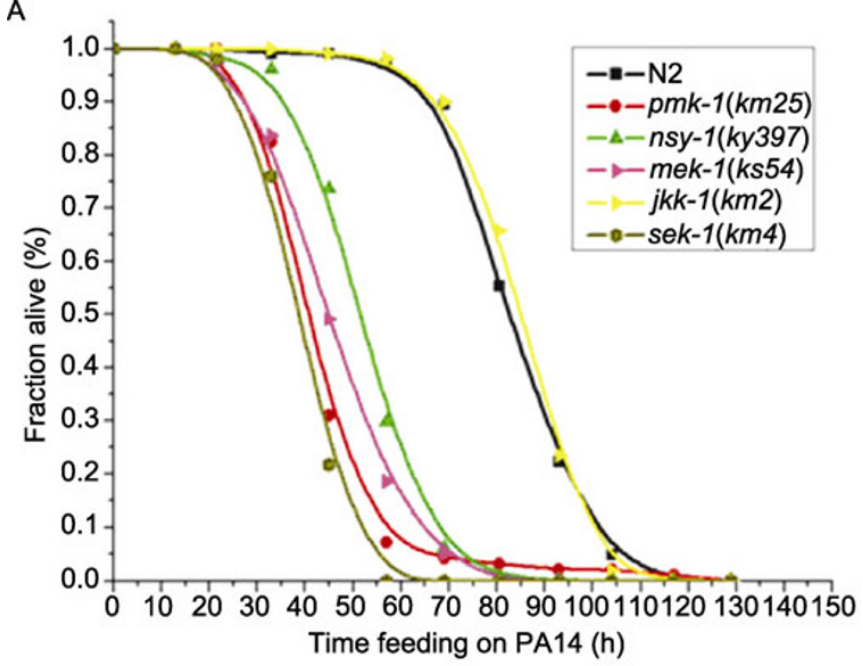

C

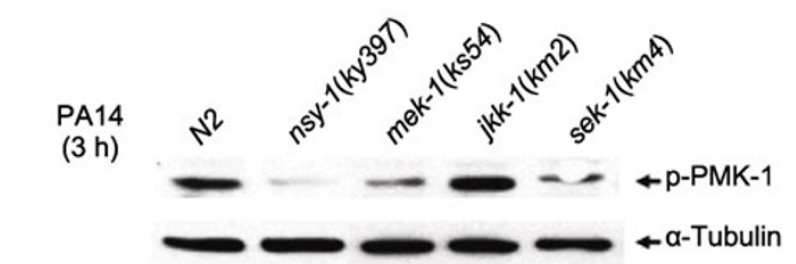

activation of p38/PMK-1 is also significantly reduced in nsy-1, sek-1 or pmk-1 mutants in response to PA 14, suggesting an involvement of NSY-1 and SEK-1 in regulating the activation of p38/PMK-1 (Figs. 2C and S2).

\section{C. elegans MAPKK MEK-1 is required in the activation of PMK-1 for resistance to $P$. aeruginosa infection}

Although NSY-1/SEK-1/PMK-1 cassette was known as an evolutionarily conserved module used in the defense against $P$. aeruginosa infection, whether other MAP3Ks or MAP2Ks also contribute to the activation of $P M K-1$, remains unclear. We found that the mutation of mek-1 also significantly increased the susceptibility of $C$. elegans to $P$. aeruginosa infection. However, there is no significant change of susceptibility in the $j k k-1$ mutants to $P$. aeruginosa infection, compared to wild type N2 worms (Fig. 2A). We have utilized RNAi to reduce the expression of mek-1 and $j k k-1$, and analyzed the susceptibility of these RNAi-treated worms to a pathogenic $P$. aeruginosa infection. As shown in Fig. 2B, the RNAi of mek-1, but not of $j k k-1$, made $C$. elegans more susceptible to $P$. aeruginosa infection. As demonstrated in previous B

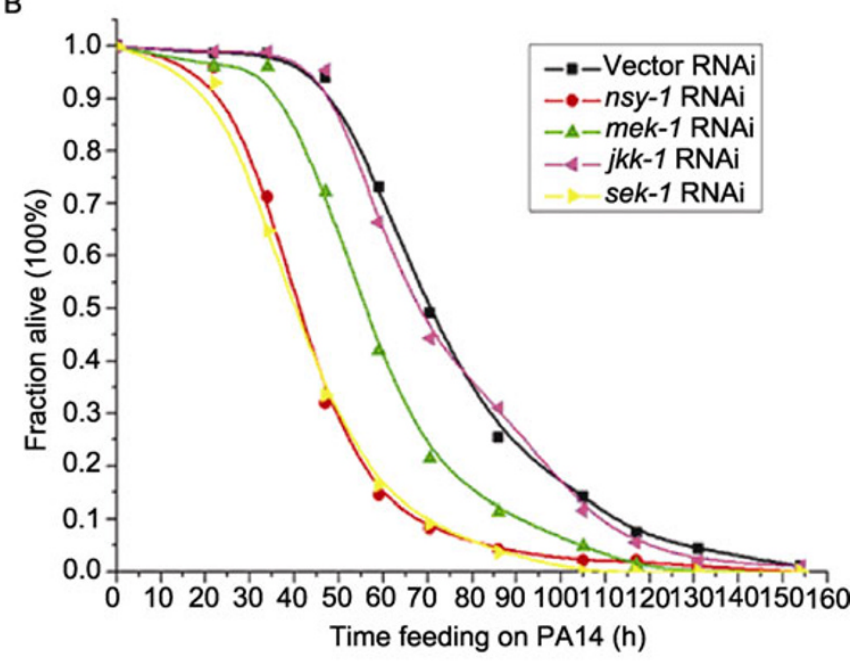

Figure 2. Activation of PMK-1 is required for resistance to $P$. aeruginosa infection. (A) Pathogen susceptibility of $C$. elegans strains N2, and mutant strains KU25 (pmk-1, km25), CX4998 (nsy-1, ky397), FK171 (mek-1, ks54), KU2 (jkk-1, km2), and KU4 (sek-1, km4) to P. aeruginosa strain PA-14. Synchronized populations of young adult worms were transferred to lawns of $P$. aeruginosa strain PA-14. Assays were performed as described under methods and materials. (B) Synchronized populations of NL2099 L1 larvae were propagated on E. coli HT115 carrying either vector control (L4440), L4440-nsy-1 positive control, or L4440-mek-1, L4440-jkk-1, L4440-sek-1, respectively, at $20^{\circ} \mathrm{C}$. They were transferred to lawns of $P$. aeruginosa strain PA-14 at the $\mathrm{L} 4$ or young adult stage and counted at time indicated. $N 2$ and mutant worms were transferred to NGM plates seeded with $P$. aeruginosa for $3 \mathrm{~h}$. (C) Worm extracts were immunoblotted with anti-phospho-p38 for the detection of PMK-1 activation (upper panel), and with anti-a-tubulin for the detection of total protein (lower panel). Data shown represent at least three independent experiments. 
research, we also found that the activation of p38/PMK-1 is significantly reduced in the mek-1 mutants in response to $P$. aeruginosa, suggesting a general involvement of MEK-1 in regulating the activation of p38/PMK-1 (Fig. 2C). In contrast, the mutation of $j k k-1$, did not cause a significant change in the p38/PMK-1 activation in response to $P$. aeruginosa infection (Fig. 2C). These results indicate that MEK-1 is required in $P$. aeruginosa pathogen resistance by activation of p38/PMK-1.

\section{C. elegans MAPKK MKK-4 is required in the activation of PMK-1 for resistance to $P$. aeruginosa infection by a DLK-1-independented manner}

Genomic data of $C$. elegans was listed out the candidate components of the JNK and p38 pathways in $C$. elegans by comparison to mammalian homologs, and corresponding $C$. elegans mutants (Brenner et al., 1974; Kawasaki et al., 1999; Koga et al., 2000; Byrd et al., 2001; Sagasti et al., 2001; Sakaguchi et al., 2004; Nakata et al., 2005; Troemel et al., 2006). To confirm that jkk-1 is not involved in a response to the $P$. aeruginosa infection, and to find out whether other MAPKKKs and MAPKKs are involved in the response to pathogenic infections, we constructed RNAi plasmids for some of these candidate genes, as listed in Table 1. Worms containing these RNAi plasmids were analyzed for their susceptibility to $P$. aeruginosa infection. As shown in Fig. 3A and $3 \mathrm{~B}$, worms treated with RNAi mkk-4, consisting of the mkk-4 mutant strain CZ4213 (ju91), showed a higher susceptibility to $P$. aeruginosa infection compared to vector treated NL2099 worms. We next investigated whether mkk-4 regulates the activation of PMK-1 in case of a $P$. aeruginosa infection. The $m k k-4$ mutant strain CZ4213 (ju91) or $m k k-4$ RNAi-treated worms were infected with $P$. aeruginosa and then analyzed for the activation of PMK-1. The activation of PMK-1 in the mkk-4 mutant strain CZ4213 (ju91) or mkk-4 RNAi-treated worms was markedly inhibited when infected with $P$. aeruginosa infection (Figs. $3 \mathrm{C}$ and S1B). However, both jkk-1 mutant strains and dlk-1 mutant strains died not significantly faster than the wild type worms, when infected with $P$. aeruginosa (Fig. 3A). Consequently, also $j k k-1$ and dlk-1 do not regulate the activation of PMK-1 in $P$. aeruginosa infection (Fig. 3C). These results suggest that $m k k-4$ influences the resistance of $C$. elegans to $P$. aeruginosa infection, by regulating the PMK-1 activation, whereas the effect is not likely to be regulated by dlk-1.

Specific MAPKKK MOM-4 is required in the activation of PMK-1 for resistance to $P$. aeruginosa infection

A previous study revealed that mammalian specific MAPKKK's TAK1 plays a very important role in the mammalian innate immune system (Ninomiya et al., 1999). However, there still exists no direct evidence of whether MOM-4, a homolog of the mammalian TAK1, participates in the innate immune response to pathogenic infections in C. elegans. To investigate whether MOM-4 is also involved in the PMK-1 activation in response to pathogenic infections, we used mom-4 mutant worms or RNAi treated worms to analyze the activation of PMK-1 in these worms, when challenged by a $P$. aeruginosa infection. As shown in Fig. 4A and 4B, mom-4 mutant worms and RNAi-treated worms exhibit a higher susceptibility to $P$. aeruginosa infection compared to the wild

Table 1. Conserved MAPK kinases that may be involved in PMK-1 activation in C. elegans and corresponding $C$. elegans mutants

\begin{tabular}{|l|l|l|l|}
\hline Class & C. elegans protein & C. elegans mutant & Mammalian homolog \\
\hline MAPKKK & NSY-1 & $k y 393$ & ASK1, ASK2 \\
\hline & DLK-1 & $j u 476$ & DLK1, LZK1 \\
\hline MOM-4 & or39 & TAK1 \\
\hline MAPKK & SEK-1 & $k m 4$ & MKK3, MKK6 \\
\hline & MKK-4 & $j u 91$ & MKK4 \\
\hline JKK-1 & $k m 2$ & MKK7 \\
\hline MEPK & MEK-1 & $k s 54$ & MKK7 \\
\hline
\end{tabular}

Candidate components in this study involved in MAPK cascades and the corresponding $C$. elegans mutants listed in table 1. Among the MAPKKs identified, JKK-1 (corresponding to gene F35C8.3) and MEK-1 (corresponding to gene K08A8.1) belong to the MKK7 subgroup. SEK-1 (corresponding to gene R03G5.2) belongs to the MKK3/6 subgroup (7). MKK-4 (corresponding to gene F42G10.2), VZC374L.1 and ZC449.3 have similarities to MKK4. Members of the MAPKKK superfamily, such as MEKK, ASK and TAK are known to be activators of the JNK and p38 MAPK cascades in mammals. The $C$. elegans genome contains an ASK homolog (nsy-1: corresponding to gene F59A6.1), a DLK/LZK homolog (dlk-1: corresponding to gene F33E2.2), a TAK1 homologs (MOM-4: corresponding to gene F52F12.3 and Y105C5A.24). Obvious homologs of MEKK1, MEKK2 and MEKK3 have not been found (Sakaguchi et al., 2004). 

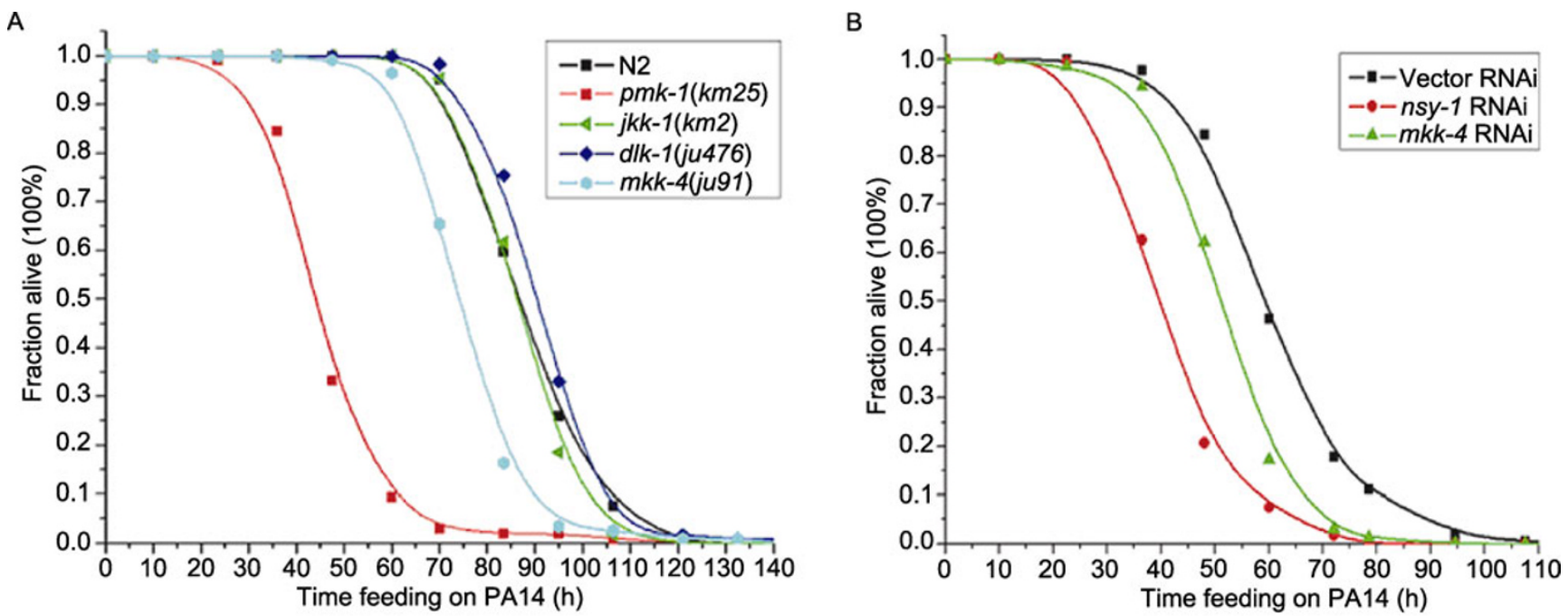

C

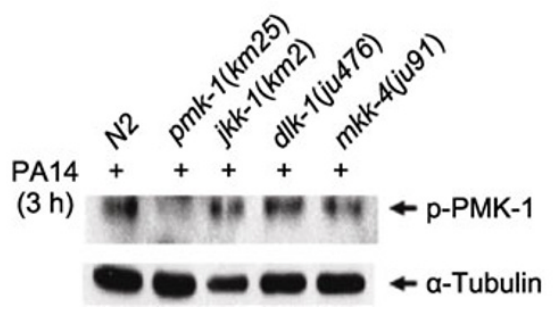

Figure 3. C. elegans MAPKK MKK-4 are required in activation of PMK-1 for resistance to $P$. aeruginosa infection by DLK-1-independented manner. (A) Susceptibility of N2, and mutant strain pmk-1(km25), jkk-1(km2), dlk-1(ju476), and mkk-4(ju91) worms to pathogenic infection ( $P$. aeruginosa $3 \mathrm{~h}$ ). (B) Synchronized populations of NL2099 L1 larvae were propagated on E. coli HT115, carrying either vector control (L4440), L4440- mkk-4 or L4440-nsy-1 control, respectively, at $20^{\circ} \mathrm{C}$. They were transferred to lawns of $P$. aeruginosa strain PA-14 at the L4 or young adult stage and counted at time indicated. (C) C. elegans strain N2, as well as mutant strain pmk-1(km25), jkk-1(km2), dlk-1(ju476), and mkk-4(ju91) worms were exposed to pathogenic infection ( $P$. aeruginosa $3 \mathrm{~h}$ ). Worm extracts were immunoblotted with anti-phospho-p38 for the detection of PMK-1 activation (upper panel), and with anti- $\alpha$-tubulin for the detection of total protein (lower panel).

type worms. Furthermore, the $P$. aeruginosa induced activation of PMK-1 markedly reduced in the mom-4 mutant worms compared to the wild type worms (Fig. 4C). This suggests that MOM-4 is required for the resistance of the worm to pathogenic infections, and that MOM-4 regulates response to $P$. aeruginosa infection by activation of $\mathrm{p38} / \mathrm{PMK}-1$.

\section{DISCUSSION}

The natural environment of $C$. elegans is soil, in which $C$. elegans is likely to encounter and feed on a diversity of pathogenic micro-organisms. It can be assumed, therefore, that this nematode has evolved a multifaceted immune response to potential pathogen infections. The p38 homolog PMK-1 pathway in C. elegans is crucial in its resistance to pathogenic infections (Berman et al., 2001; Kim et al., 2002, 2005). In this study, we have found that the PMK-1 pathway of $C$. elegans can be activated under heat, UV, Sorbitol and $P$. aeruginosa stress. We, furthermore, confirmed that the
NSY $-1 \rightarrow$ SEK- $1 \rightarrow$ PMK-1 cascade is involved in the response to these stresses. In addition, this study revealed that the MEK-1, same as the SEK-1 upstream signaling molecular pathway of PMK-1, also regulates the PMK-1 activation in response to heat, UV, Sorbitol and $P$. aeruginosa stress (data not shown).

C. elegans is particularly suited for reverse genetic analyses, since loss-of-function RNAi phenotypes can be generated efficiently by feeding the worms with bacteria expressing double-stranded RNA (dsRNA) that is homologous to a target gene (Kamath et al., 2003). In addition, genomic RNAi clones are readily available commercially. As the homologs of MAPKs in mammals are already known from the existing genomic sequence data (Sakaguchi et al., 2004), we performed a reverse screening for new MAP kinases that could be involved in the resistance of $C$. elegans to pathogens. MKK-4, which is the homolog of mammalian MKK-4, was found to be required for $P$. aeruginosa pathogen resistance. However, the activation of PMK-1 in $m k k-4$ RNAi 
A

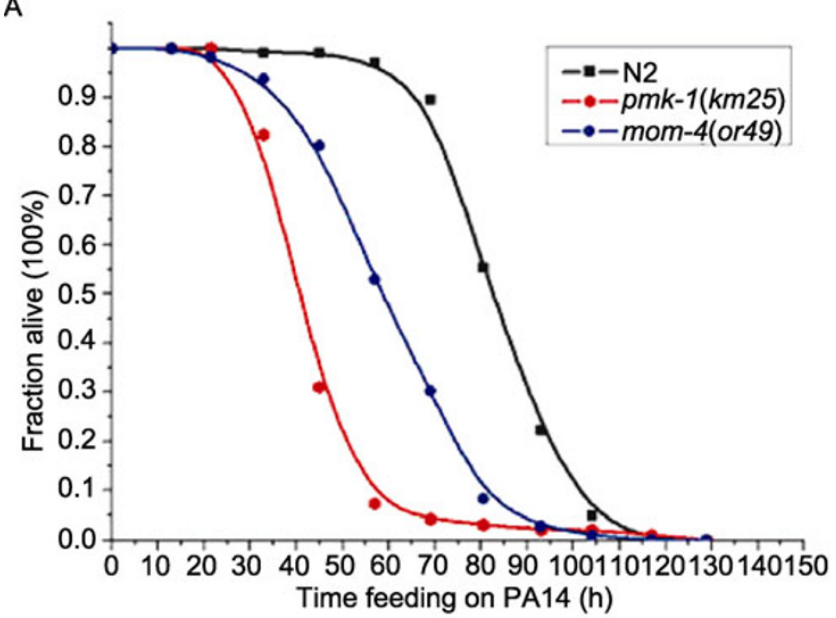

B

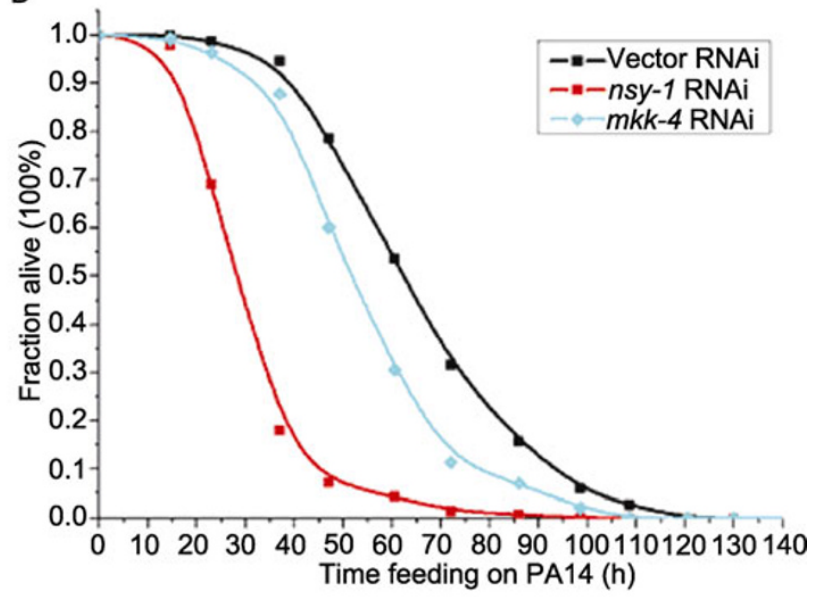

C

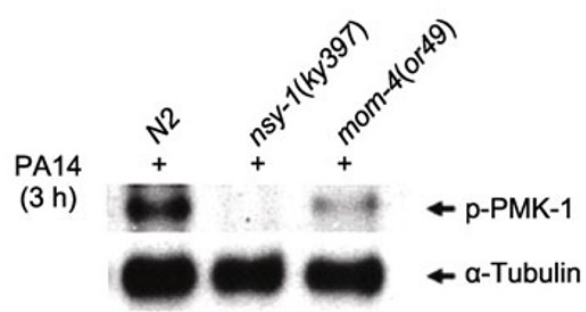

Figure 4. Specific MAPKKKs MOM-4 specifically regulate the PMK-1 activation under $P$. aeruginosa infection. $C$. elegans strain N2, and mutant strains CX4998 (nsy-1, ky397), EU446 (mom-4, or49) were treated with pathogenic infection (P. aeruginosa $3 \mathrm{~h}$ ). Either pmk-1 mutants for control (A) or synchronized populations of NL2099 L1 larvae were propagated on E. coli HT115 that either carried vector control (L4440)L4440-mom-4, as well as $L 4440-n s y-1$ positive control (B) at $20^{\circ} \mathrm{C}$. They were transferred to lawns of $P$. aeruginosa strain PA-14 at the L4 or young adult stage and counted at time indicated. (C) Worm extracts were immunoblotted with anti-phospho-p38 for the detection of PMK-1 activation (upper panel), and with anti-a-tubulin for the detection of total protein (lower panel).

treated $N 2$ worms was not regulated by dlk-1 when infected by $P$. aeruginosa. This suggests that in the innate immune response of $C$. elegans mkk-4 may activate PMK-1, but by DLK-1 independent signaling pathways. These results suggested that MKK4 may be involved in the resistance of $C$. elegans to pathogens by p38 pathways, but not by the JNK pathway, which concurs with previous findings (Brancho et al., 2003). Further studies are needed to substantiate the upstream MAP kinases MKK4, and how they are involved in the innate immune response to $P$. aeruginosa infection in $C$. elegans.

Our study is the first to report that the nematode homolog of TGF- $\beta$ kinase-1 (TAK-1), MOM-4 is important for worm survival under $P$. aeruginosa pathogenic stress in $C$. elegans. Previous studies demonstrated that TAK1 plays important roles in immunological and developmental contexts across many species (Plowman et al., 1999; Sakaguchi et al., 2004; Wan et al., 2006; Delaney et al., 2006; Kajino et al., 2008). In mammalian tissues, TAK1 is essential for responses to a variety of inflammatory ligands, it can involve in p38 activation by MKK3/6 or in JNK activation by MKK4/7 (Kim et al., 2005). The innate immune responses of Drosophila require TAK1 for the expression of a number of antimicrobial pep- tides. Further study find that mom- 4 carries out these diverse biological roles through a number of signaling pathways, such as the TGF- $\beta / B M P, W n t / F z, J N K$ and NF-KB pathways (Delaney et al., 2006). The present study found that mom-4 regulate the PMK-1 pathway in the innate immune response to pathogenic infections in C. elegans, while the actual function of TAK-1 in the PMK-1 in C. elegans remains unexplained, TAB-1 may be involved in it (Lu et al., 2006). Despite the fact that there still is no experimental data available to prove the direct interaction between mom- 4 and sek-1, killing assays of RNAi treated worms revealed that mom- 4 might be functionally dependent on sek-1 in pathogenic infections (Fig. $4 \mathrm{~A}$ and $4 \mathrm{~B})$.

The NSY-1 $\rightarrow$ SEK-1 $\rightarrow$ PMK-1 pathway plays a central role in the innate immune response to pathogenic infections. In this study we identified MOM-4 is involved in the resistance of $C$. elegans, to $P$. aeruginosa infection through regulating PMK-1 activation aside from the conserved NSY-1/ SEK-1/PMK-1 cassette. MKK-4, another MAP2K, also showed similar roles. However, MEK-1 and JKK-1, both are mammalian MKK7 homolog, showed different effects, MEK-1 also regulated the PMK- 1 activation in the $P$. aeruginosa infection, but JKK-1 was not functional. So we surmise that 
MKK-4 could be the upstream kinase of PMK-1 in the case of a $P$. aeruginosa infection in a DLK-1-independent manner.

\section{MATERIALS AND METHODS}

Worm strains and culture

Strains N2, NL2099 rrf-3 (pk1426) II, nsy-1(ky397) II, CZ4213 mkk-4(ju91) X, CZ5730 dlk-1(ju476) I, mom-4(or39) I/hT2 (l;IIl) ( Ninomiya et al.,1999), FK171 mek-1(ks54) X, KU2 jkk-1(km2) X, KU4 sek-1(km4) X, KU25 pmk-1(km25) IV, and TJ1052 age-1(hx546)Il were obtained from the Caenorhabditis Genetics Center (CGC). Nematodes were handled using standard methods with a few modifications (Brenner et al., 1974). We used the E. coli OP50 streptomycin resistant variant strain OP50-1 as the food source for the worm strains to avoid possible contamination with other microbes (Shapira et al., 2006). OP50-1 also obtained from CGC. The $P$. aeruginosa PA-14 strain was kindly provided by Dr. Ausubel's lab at the Massachusetts General Hospital, Harvard School of Medicine.

\section{Bacteria-mediated RNAi}

The bacteria-mediated RNAi process was carried out as described in references (Kamath et al., 2003; Timmons et al.,2001). Eggs from bleach synchronized gravid adult worms were hatched in M9 buffer at $20^{\circ} \mathrm{C}$ for $12 \mathrm{~h}$. Next, the L1 worms (early larval stage) were transferred to the RNAi plates seeded with the E. coli strain HT115. HT115 had been transformed with $L 4440$ vector or specific genomic DNA fragments in advance. The RNAi plates consisted of $1 \mathrm{mmol} / \mathrm{L}$ IPTG in addition to the $50 \mu \mathrm{g} / \mathrm{mL}$ Ampicilin in the NGM plates. The worms were grown in the RNAi plates to $\mathrm{L} 4$ or young adult stage at $20^{\circ} \mathrm{C}$, and then used in the assays. Genomic DNA fragments of specific genes were cloned using primers named in the WormBase list of $C$. elegans genomic clones (www.wormbase.org). The primers used in this study are: $m k k-4$, F: atcgctgcagACGCAATTCAATGTCCCTTC, R: actgaagcttTCGTCGCTGTCTGGATGTAG; mom-4, F: atcgccatggTAAATTCAGACACCAACACAACG, R: actgctgcagAAGTCGGTCGAATATCAGCATTA; mlk-1, F: atcgcccgggTGCGTTGCACTTTTCATCTC, R: actgctgcagGTTAACGCTTTGCAGGAAGC; $n s y-1$, F: atcgctgcagTGAAGCAGCTTTGATGATGG, R: actgaagcttTTCGGTTACTGGATTCAGCC; sek-1, F: atcgctgcagCCGAGACTTAAGCAAATCGC, R: actgaagcttGCTTCTCATTTCTTGCCTGC; $j k k-1$, F: atcgctgcagTGAGATACACATCCGGACCA; R: actgaagcttGAACCAATCCGCAACTTGAT; mek-1, F: atcgccatggGAAGGAGCTTCAGTTCGTGG, R: actgctcgagCTCGACGTCGGTTTCAATTT; unc-22, F: actgctgcagCACTCTTACTGCTACCAACGCTT, R: atcgccatggAATGATCTCCCTTGTTGAGTGAA.

The DNA fragments were cloned into $L 4440$ plasmids and transformed into HT115 for RNAi experiments as previously described (Timmons et al., 2001; Kamath et al., 2003). We used unc-22 as the control to observe RNAi efficiency, RNAi treated worms washed with M9 buffer, and then used for subsequent assays.

Worm stress and life span assays

Slow killing $P$. aeruginosa assays were performed using the $P$. aeruginosa strain as described (Tan et al., 1999). All assays performed using L4 or young adult stage hermaphrodites, unless noted.
Briefly, P. aeruginosa was cultured in King's B broth, seeded on slow-kill plates, which contain NGM $(0.35 \%$ instead of $0.25 \%$ peptone), and was subsequently incubated for $24 \mathrm{~h}$ at $37^{\circ} \mathrm{C}$ and then for another $24 \mathrm{~h}$ at $25^{\circ} \mathrm{C}$ before adding the worms. $100 \mu \mathrm{g} / \mathrm{mL}$ of 5-fluorodeoxyuridine (FUDR, Hisun Pharmaceutical Co. Ltd.) was added to the NGM killing plates to prevent progeny from hatching (Kim et al., 2002). A total of 30-50 L4 or young adult stage worms were transferred to each pathogen plate. Three plates per strain were tested for each experiment.

For the heat stress assays, young adult $\mathrm{N} 2$ worms were washed with M9 buffer from OP50-1 NGM plates, and incubated in a $35^{\circ} \mathrm{C}$ water bath for the time indicated.

For osmotic stress assays, sorbitol was added to young adult N2 worms in M9 buffer to a final concentration of $200 \mathrm{mmol} / \mathrm{L}$, and incubated for the time indicated.

UV stress assays were performed using $1200 \mathrm{~J}$ UVB (HL-2000 HybriLinker, UVP, LLC, USA), as described (Wolff et al., 2006).

For all four modes of stress, the treated worms were counted every $6-12 \mathrm{~h}$ and scored by gentle prodding using a platinum wire. They were scored as dead without response after at least 3 gentle proddings. All assays were conducted at $25^{\circ} \mathrm{C}$. Three plates per strain were tested for each experiment.

\section{Life span assays}

Life span assays were performed as described in references (Berman et al., 2001; Troemel et al., 2006). Gravid adult worms were synchronized by bleach method. Eggs were hatched and grown to L4 or young adult stage on NGM plates at $20^{\circ} \mathrm{C}$. About $20-30$ animals were then transferred to each NGM plate seeded with OP50-1. Subsequently, $100 \mu \mathrm{g} / \mathrm{mL}$ of 5-fluorodeoxyuridine (FUDR) was added to the NGM plates. The assays were performed at $25^{\circ} \mathrm{C}$. Three plates per strain were tested for each experiment. Worms were scored every 2 days by gentle prodding using a platinum wire to test for live or dead animals. Origin7.5 software was used to calculate the killing assay and life span data, as well as to produce the graphs for this study.

\section{SDS-PAGE and immunoblot analysis}

Immunoblot analyses were performed as described in reference (Kim et al., 2002), with a few modifications. Worms subjected to various stresses were lysed in $2 \times$ Laemmli sample buffer to which cocktail inhibitors were added (Sigma). They were instantly frozen in liquid N2. Samples were then boiled at $100^{\circ} \mathrm{C}$ for $10 \mathrm{~min}$. Total protein of each sample was determined by BCA kit (Beyotime Co. Ltd.). The same amount of protein for each sample was resolved by SDS-PAGE $(10 \%$ gels) and transferred to nitrocellulose membranes. The membranes were blocked with $1 \%$ gelatin and subjected to antibody incubation. Antibodies that recognize doubly phosphorylated p38 (Biosource) for PMK-1 detection, and a-tubulin (Sigma) were used for total protein as loading control (Tanaka et al., 2002).

\section{ACKNOWLEDGEMENTS}

We thank Professor Dennis H. Kim of MIT, Boston, USA, for his technical advice. We furthermore thank Professor Frederick Ausubel of Harvard Medical School, Boston, USA, for providing us with the $P$. aeruginosa strain. We are grateful to $\mathrm{Dr}$. Pu Pu of the Shanghai Institute for Biological Sciences for furnishing us with his valuable technical 
assistance. This project was This work was supported by the National Basic Research Program (973 Program) (Nos.2012CB578100 and 2011CB505000), National Natural Science Foundation of China (Grant No. 31030028), the Science and Technology Commission of Shanghai Municipality (Program 10JC1416400 and 12XD1406700), the Innovation Program of Shanghai Municipal Education Commission (Project 12ZZ117) and from Cross-fund of Biomedical Engineering of Shanghai Jiaotong University.

\section{ABBREVIATIONS}

ASK-1, apoptosis signaling-regulating kinase-1; DLK-1, DAP like kinase; JKK, JNK kinase; LZK, Leucine zipper-bearing kinase; MEK-1, MAP kinase kinase or ERK kinase-1; MEKK, Mitogen-activated protein/ERK kinase kinase; MLK, Mixed lineage kinase; MKK, MAP kinase kinase; MOM-4, More of MS-4; NSY-1, neuronal symmetry-1; PMK-1, p38 MAP kinase family; SEK-1, SAPK/ERK kinase-1; TAK, Transforming growth factor beta activated kinase

\section{REFERENCES}

Berman, K., McKay, J., Avery, L., and Cobb, M. (2001). Isolation and characterization of pmk-(1-3): three p38 homologs in Caenorhabditis elegans. Mol Cell Biol Res Commun 4, 337-344.

Brancho, D., Tanaka, N., Jaeschke, A., Ventura, J.J., Kelkar, N., Tanaka, Y., Kyuuma, M., Takeshita, T., Flavell, R.A., and Davis R.J. (2003). Mechanism of p38 MAP kinase activation in vivo. Genes Dev 17, 1969-1978.

Brenner, S. (1974). The genetics of Caenorhabditis elegans. Genetics 77, 71-94.

Byrd, D.T., Kawasaki, M., Walcoff, M., Hisamoto, N., Matsumoto, K., and Jin, Y. (2001). UNC-16, a JNK-signaling scaffold protein, regulates vesicle transport in C. elegans. Neuron 32, 787-800.

Chiariello, M., Marinissen M.J., and Gutkind J.S. (2000). Multiple mitogen-activated protein kinase signaling pathways connect the cot oncoprotein to the c-jun promoter and to cellular transformation. Mol Cell Biol 20, 1747-1758.

Cobb, M.H., Boulton T.G., and Robbins D.J. (1991). Extracellular signal-regulated kinases: ERKs in progress. Cell Regul 2, 965-978.

Deacon, K. and Blank J.L. (1999). MEK kinase 3 directly activates MKK6 and MKK7, specific activators of the p38 and c-Jun NH2-terminal kinases. J Biol Chem. 274(23), 16604-16610.

Deak, M., Clifton, A.D., Lucocq, L.M., and Alessi, D.R. (1998). Mitogen- and stress-activated protein kinase-1 (MSK1) is directly activated by MAPK and SAPK2/p38, and may mediate activation of CREB. EMBO J 17, 4426-4441.

Delaney, J.R. and Mlodzik, M., (2006).TGF-beta activated kinase-1: new insights into the diverse roles of TAK1 in development and immunity. Cell Cycle 5, 2852-2825.

Dérijard, B., Hibi, M., Wu, I.H., Barrett, T., Su, B., Deng, T., Karin, M., and Davis, R.J. (1994). JNK1: a protein kinase stimulated by UV light and Ha-Ras that binds and phosphorylates the c-Jun activation domain. Cell 76, 1025-1037.

Fukunaga, R. and Hunter T. (1997). MNK1, a new MAP kinase-activated protein kinase, isolated by a novel expression screening method for identifying protein kinase substrates. EMBO J 16, 1921-1933.
Han, J., Lee, J.D., Bibbs, L., and Ulevitch, R.J. (1994). A MAP kinase targeted by endotoxin and hyperosmolarity in mammalian cells. Science 265, 808-811.

Han, J., Jiang, Y., Li, Z., Kravchenko, V.V., and Ulevitch, R.J. (1997). Activation of the transcription factor MEF2C by the MAP kinase p38 in inflammation. Nature 386, 296-299.

Herskowitz, I. (1995). MAP kinase pathways in yeast: for mating and more. Cell 80, 187-197.

Ichijo, H., Nishida, E., Irie, K., ten Dijke, P., Saitoh, M., Moriguchi, T., Takagi, M., and Matsumoto, K. (1997). Induction of apoptosis by ASK1, a mammalian MAPKKK that activates SAPK/JNK and p38 signaling pathways. Science 275, 90-94.

Iordanov, M., Bender, K., Ade, T., Schmid, W., Sachsenmaier, C., Engel, K., Gaestel, M., Rahmsdorf, H.J., and Herrlich, P. (1997). CREB is activated by UVC through a p38/HOG-1-dependent protein kinase. EMBO J 16, 1009-1022.

Kajino-Sakamoto, R., Inagaki, M., Lippert, E., Akira, S., Robine, S., Matsumoto, K., Jobin, C., and Ninomiya-Tsuji, J. (2008). Enterocyte-derived TAK1 signaling prevents epithelium apoptosis and the development of ileitis and colitis. J Immunol 181, 1143-1152.

Kamath, R.S., Fraser, A.G., Dong, Y., Poulin, G., Sohrmann, M., Welchman, D.P., Zipperlen, P., and Ahringer, J. (2003). Systematic functional analysis of the Caenorhabditis elegans genome using RNAi. Nature 421, 231-237.

Kawasaki, M., Hisamoto, N., lino, Y., Yamamoto, M., Ninomiya-Tsuji, J., and Matsumoto, K. (1999). A Caenorhabditis elegans JNK signal transduction pathway regulates coordinated movement via type-D GABAergic motor neurons. EMBO J 18, 3604-3615.

Kim, D.H. and Ausubel F.M. (2005). Evolutionary perspectives on innate immunity from the study of Caenorhabditis elegans. Curr Opin Immunol 17, 4-10.

Kim, D.H., Feinbaum, R., Alloing, G., Emerson, F.E., Garsin, D.A., Inoue, H., Tanaka-Hino, M., Hisamoto, N., Matsumoto, K., Tan, M.W., et al. (2002). A conserved p38 MAP kinase pathway in Caenorhabditis elegans innate immunity. Science 297,623-626.

Koga, M., Zwaal, R., Guan, K.L., Avery, L., and Ohshima, Y. (2000). A Caenorhabditis elegans MAP kinase kinase, MEK-1, is involved in stress responses. EMBO J 19, 5148-5156.

Kyriakis, J.M. and Avruch, J. (2001). Mammalian mitogen-activated protein kinase signal transduction pathways activated by stress and inflammation. Physiol Rev 81, 807-869.

Kyriakis, J.M., Banerjee, P., Nikolakaki, E., Dai, T., Rubie, E.A., Ahmad, M.F., Avruch, J., and Woodgett, J.R. (1994).The stress-activated protein kinase subfamily of c-Jun kinases. Nature 369, 156-160.

Lee, J.C., Laydon, J.T., McDonnell, P.C., Gallagher, T.F., Kumar, S., Green, D., McNulty, D., Blumenthal, M.J., Heys, J.R., and Landvatter, S.W. (1994). A protein kinase involved in the regulation of inflammatory cytokine biosynthesis. Nature 372, 739-746.

Lu, G., Kang, Y.J., Han, J., Herschman, H.R., Stefani, E., and Wang, Y. (2006). TAB-1 modulates intracellular localization of p38 MAP kinase and downstream signaling. J Biol Chem 281, 6087-6095.

Nakata, K., Abrams, B., Grill, B., Goncharov, A., Huang, X., Chisholm, A.D., and Jin, Y. (2005). Regulation of a DLK-1 and p38 MAP kinase pathway by the ubiquitin ligase RPM-1 is required for presynaptic development. Cell 120, 407-420.

New, L., Jiang, Y., Zhao, M., Liu, K., Zhu, W., Flood, L.J., Kato, Y., 
Parry, G.C., and Han, J. (1998). PRAK, a novel protein kinase regulated by the p38 MAP kinase. EMBO J 17, 3372-3384.

Ninomiya-Tsuji, J., Kishimoto, K., Hiyama, A., Inoue, J., Cao, Z., and Matsumoto, K. (1999). The kinase TAK1 can activate the NIK-I kappaB as well as the MAP kinase cascade in the IL-1 signalling pathway. Nature 398, 252-256.

Pierrat, B., Correia, J.S., Mary, J.L., Tomás-Zuber, M., and Lesslauer, W. (1998). RSK-B, a novel ribosomal S6 kinase family member, is a CREB kinase under dominant control of p38alpha mitogen-activated protein kinase (p38alphaMAPK). J Biol Chem. 273, 29661-29671.

Plowman, G.D., Sudarsanam, S., Bingham, J., Whyte, D., and Hunter, T. (1999).The protein kinases of Caenorhabditis elegans: a model for signal transduction in multicellular organisms. Proc Natl Acad Sci U S A 96, 13603-13610.

Raingeaud, J., Whitmarsh, A.J., Barrett, T., Derijard, B, and Davis, R.J. (1996). MKK3- and MKK6-regulated gene expression is mediated by the p38 mitogen-activated protein kinase signal transduction pathway. Mol Cell Biol 16, 1247-1255.

Sagasti, A., Hisamoto, N., Hyodo, J., Tanaka-Hino, M., Matsumoto, K., and Bargmann, C.I. (2001). The CaMKII UNC-43 activates the MAPKKK NSY-1 to execute a lateral signaling decision required for asymmetric olfactory neuron fates. Cell 105, 221-232.

Sakaguchi, A., Matsumoto K., and Hisamoto N. (2004). Roles of MAP kinase cascades in Caenorhabditis elegans. J Biochem (Tokyo) 136, 7-11.

Schaeffer, H.J. and Weber M.J. (1999).Mitogen-activated protein kinases: specific messages from ubiquitous messengers. Mol Cell Biol 19, 2435-2444.

Shapira, M., Hamlim, B., Rong, J.M., Chen, K., Ronen, M., and Tan, M.W. (2006). A conserved role for a GATA transcription factor in regulating epithelial innate immune responses. Proc Natl Acad Sci U S A 103, 14086-14091.

Shivers, R.P., Pagano, D.J., Kooistra, T., Richardson, C.E., Reddy, K.C., Whitney, J.K., Kamanzi, O., Matsumoto, K., Hisamoto, N., and Kim, D.H. (2010). Phosphorylation of the conserved transcription factor ATF-7 by PMK-1 p38 MAPK regulates innate immunity in Caenorhabditis elegans. PLoS Genet 6, e1000892

Stokoe, D., Campbell, D.G., Nakielny, S., Hidaka, H., Leevers, S.J., Marshall, C., and Cohen, P. (1992). MAPKAP kinase-2; a novel protein kinase activated by mitogen-activated protein kinase. EMBO J 11, 3985-3994.

Symons, A., Beinke S., and Ley S.C. (2006). MAP kinase kinase kinases and innate immunity. Trends Immunol 27, 40-48.

Tan, M.W., Rahme, L.G., Sternberg, J.A., Tompkins, R.G., and Ausubel, F.M. (1999). Pseudomonas aeruginosa killing of Caenorhabditis elegans used to identify $P$. aeruginosa virulence factors. Proc Natl Acad Sci U S A 96, 2408-2413.
Tanaka, N., Kamanaka, M., Enslen, H., Dong, C., Wysk, M., Davis, R.J., and Flavell, R.A. (2002). Differential involvement of p38 mitogen-activated protein kinase kinases MKK3 and MKK6 in T-cell apoptosis. EMBO Rep 3, 785-791.

Tibbles, L.A., Ing, Y.L., Kiefer, F., Chan, J., Iscove, N., Woodgett, J.R., and Lassam, N.J. (1996). MLK-3 activates the SAPK/JNK and p38/RK pathways via SEK1 and MKK3/6. EMBO J 15, 7026-7035.

Timmons, L., Court D.L., and Fire A. (2001). Ingestion of bacterially expressed dsRNAs can produce specific and potent genetic interference in Caenorhabditis elegans. Gene 263,103-112.

Troemel, E.R., Chu, S.W., Reinke, V., Lee, S.S., Ausubel, F.M., Kim, D.H. (2006). p38 MAPK regulates expression of immune response genes and contributes to longevity in C. elegans. PLoS Genet 2, 1725-1739.

Wan, Y.Y., Chi, H., Xie, M., Schneider, M.D., and Flavell, R.A. (2006). The kinase TAK1 integrates antigen and cytokine receptor signaling for T cell development, survival and function. Nat Immunol 7, 851-858.

Wang, X.Z. and Ron D. (1996). Stress-induced phosphorylation and activation of the transcription factor CHOP (GADD153) by p38 MAP Kinase. Science 272, 1347-1349.

Waskiewicz, A.J. and Cooper J.A. (1995). Mitogen and stress response pathways: MAP kinase cascades and phosphatase regulation in mammals and yeast. Curr Opin Cell Biol 7, 798-805.

Waskiewicz, A.J., Flynn, A., Proud, C.G., and Cooper, J.A. (1997). Mitogen-activated protein kinases activate the serine/threonine kinases Mnk1 and Mnk2. EMBO J 16, 1909-1920.

Whitmarsh, A.J., Yang, S.H., Su, M.S., Sharrocks, A.D., and Davis, R.J. (1997). Role of p38 and JNK mitogen-activated protein kinases in the activation of ternary complex factors. Mol Cell Biol 17, 2360-2371.

Widmann, C., Gibson, S., Jarpe, M.B., and Johnson, G.L. (1999). Mitogen-activated protein kinase: conservation of a three-kinase module from yeast to human. Physiol Rev 79, 143-180.

Wolff, S., Ma, H., Burch, D., Maciel, G.A., Hunter, T., and Dillin, A. (2006). SMK-1, an essential regulator of DAF-16-mediated longevity. Cell 124, 1039-1053.

Wysk, M., Yang, D.D, Lu, H.T., Flavell, R.A., and Davis, R.J. (1999). Requirement of mitogen-activated protein kinase kinase 3 (MKK3) for tumor necrosis factor-induced cytokine expression. Proc Natl Acad Sci U S A 96, 3763-3768.

Yamaguchi, K., Shirakabe, K., Shibuya, H., Irie, K., Oishi, I., Ueno, N., Taniguchi, T., Nishida, E., and Matsumoto, K. (1995). Identification of a member of the MAPKKK family as a potential mediator of TGF-beta signal transduction. Science 270, 2008-2011.

Zhuang, Z.H., Sun, L., Kong, L., Hu, J.H., Yu, M.C., Reinach, P., Zang, J.W., and Ge, B.X. (2006). Regulation of Drosophila p38 activation by specific MAP2 kinase and MAP3 kinase in response to different stimuli. Cell Signal 18, 441-448. 